Volume 4, Issue 1 Janaury-March 2019, Pages: 274, DOI: http://dx.doi.org/10.19082/ah274

\title{
ACUTE PANCREATITIS INDUCED BY ANDROCTONUS AUSTRALIS HECTOR VENOM: INFLAMMATORY RESPONSE AND TISSUE DAMAGE
}

\author{
ZahidaTaibi-Djennah $^{1}$, Fatima Laraba-Djebari ${ }^{1}$ \\ flaraba@hotmail.com
}

USTHB, Faculty of Biological Sciences, Laboratory of Cellular and Molecular Biology, BP 32 El-Alia, Bab Ezzouar, 16111 Algiers, Algeria

\section{TYPE OF ARTICLE: CONFERENCE ABSTRACT}

\begin{abstract}
Background: Scorpion envenomation (SE) is a public health problem raging not only in Maghreb regions but also in the rest of the world. In Algeria, Androctonus australis hector (Aah) is responsible for serious and fatal accidents in humans. Complexity of biological changes and disturbances caused by scorpion venoms is multifactorial. Acute pancreatitis is an inflammatory disorder characterized by excessive recruitment of leukocytes and massive release of inflammatory mediators including TNF- $\alpha$, IL-1 $\beta$, IL- 6 . Acinar cell injury in acute pancreatitis leads to a systemic inflammatory response syndrome and multiple organ dysfunctions. Thus, multiple visceral dysfunctions observed after scorpion stings could be related to acute pancreatitis.

Objective: The aim of this study was to determine the effects of Aah venom on pancreatic function and its consequences on inflammatory response and redox status.

Methods: NMRI mice were injected by the subcutaneous route with a sublethal dose of Aah venom. Inflammatory response and oxidative stress were assessed in pancreatic tissue homogenates.

Results: The obtained results revealed that Aah venom induced inflammatory response characterized by significant increase of microvascular permeability, Matrix Metalloproteinases, NF- $\kappa$ B, Myeloperoxidase and Eosinophil Peroxidase activities in pancreatic tissue homogenates. It also induced severe alterations in pancreatic tissue associated with a significant increase in levels of nitric oxide, hydrogen peroxide and lipid peroxidation. Cytokine antagonists injected 30 minutes prior to Aah venom allowed a significant reduction of pathological biomarker of pancreatic function, inflammatory response and oxidative stress.

Conclusion: In conclusion, the Aah venom seems to provoke acute pancreatitis that contributes to systemic inflammatory response observed during SE. The pathophysiology of these events is complex; it seems to be mediated by the massive release of IL- 6 and TNF- $\alpha$.
\end{abstract}

KEYWORDS: Aah venom, Acute pancreatitis, Inflammatory response, IL-6, TNF- $\alpha$

\footnotetext{
Abstracts of Third International Conference on Health Sciences and Medical Technologies, October 2018, Tlemcen, Algeria (ICHSMT-18)

(C) 2019 The Authors. This is an open access article under the terms of the Creative Commons Attribution-NonCommercialNoDerivs License, which permits use and distribution in any medium, provided the original work is properly cited, the use is non-commercial and no modifications or adaptations are made.
} 